\title{
Swarm Stability Analysis of High-Order Linear Time-Invariant Singular Multiagent Systems
}

\author{
Mingyu Fu, ${ }^{1}$ Shimin Wang, ${ }^{1}$ and Yirui Cong ${ }^{2}$ \\ ${ }^{1}$ Departemnt of Control Theory, College of Automation, Harbin Engineering University, Harbin 150001, China \\ ${ }^{2}$ Departemnt of Control Theory, College of Automation, Northeastern University, Shenyang 110000, China
}

Correspondence should be addressed to Shimin Wang; wangshimin@hrbeu.edu.cn

Received 5 May 2013; Revised 15 June 2013; Accepted 24 June 2013

Academic Editor: Dumitru Baleanu

Copyright (c) 2013 Mingyu Fu et al. This is an open access article distributed under the Creative Commons Attribution License, which permits unrestricted use, distribution, and reproduction in any medium, provided the original work is properly cited.

\begin{abstract}
The swarm stability problem of high-order linear time-invariant (LTI) singular multiagent systems with directed graph topology is investigated extensively. Consensus of multiagent systems can be regarded as a specific case of swarm stability problem. Necessary and sufficient conditions for both swarm stability and consensus are presented. These conditions depend on the graph topology and generalized inverse theory, the dynamics of agents, and interaction among the neighbours. Several examples to illustrate the effectiveness of theoretical results are given.
\end{abstract}

\section{Introduction}

Recently the study of multiagent systems has attracted considerable attention from biologists (see $[1,2])$, physical scientists (see [3]), and engineers (see [4-8]). Due to its broad application in many areas such as satellites, spacecrafts (see [4]), aircrafts (see [5]), mobile robots, UAV, AUV (see [6]), flocking of social insects (see [7]), and design of sensor networks (see [8]). Stability is usually a basic requirement for a control system. Stability of multiagent systems is special because of the configuration difference between these systems and isolated dynamic systems. Models of these systems originated from bioscience. Studies on them have aroused great interest in the field of automatic control theory since about a decade ago. The research on stability problems of multiagent systems has been studied extensively for years. Researchers have endeavoured to give criteria for checking whether a specific multiagent system is stable. For example, Jin et al. [9] paid attention to the stability of a simple swarm system rather early. The background of their research came from engineering systems such as multiagent supporting system [10]. Based on the work of Jin, Liu et al. [11, 12] tried to extend the research to more general and critical situations, and they studied the so-called asynchronous swarm systems. However, the technical setting of their model is complicated, and the results are restrictive. Compared with the research of
Liu et al., Gazi and Passino [13, 14] and Gazi [15] studied the stability problem of some first-order models abstracted from biological systems. Chu et al. [16] extended the discussions in [13] to certain anisotropic model. Recently, Li [17] extended the results in [13] to the situation with general graph topology instead of a complete graph. Cai et al. [18] studied the swarm stability of high-order linear time-invariant multiagent systems in 2011. Besides, there were also other studies related to the stability of swarm systems, for example, the study about string stability $[19,20]$. Zhou et al. [21] studied the stability of switched linear singular systems.

Consensus of multiagent systems can be regarded as a specific case of swarm stability problem; our results shows that consensus achievement is a specific case of swarm stability, which will be called asymptotic swarm stability. Consensus problems for multiagent systems have been studied by lots of researchers. Vicsek et al. [3] proposed a discrete-time first-order model of $n$ agents moving in the horizontal plane with different headings. The systems can achieve consensus on heading via updating their heading according to their neighbor's heading. Jadbabaie et al. [22] used graph theory to give an explanation. Olfati-Saber and Murray [23] showed that the first-order consensus problems can be solved if the topology was strongly connected. Ren and Beard [24] further proved that the first-order consensus can be achieved if interaction graphs have a directed spanning tree. Xiao et al. [25] 
studied the general first-order consensus problems multiagent discrete-time systems with fixed topology. Several dynamic consensus algorithms for second-order multiagent systems and sufficient conditions for state consensus of systems have been proposed in [26, 27]. Tian and Zhang [28] discussed the high-order consensus of heterogeneous multiagent systems. Zheng and Wang [29] investigated finite-time consensus problems of heterogeneous multiagent systems. Xiao and Wang [30] have investigated the consensus problem for high-order general nonsingular multiagent systems based on the structure of certain high-dimensional matrices. Cheng et al. [31] solved a modified consensus problems of high-order normal multiagent systems with fixed fixed communication topologies. Xi et al. [32] studied the consensus problems for general high-order LTI multiagent systems which have timevarying consensus function.

Recently, more attention has been paid to the consensus problem for the singular multiagent systems. It is well known that singular systems can better describe physical systems than normal ones (see [33]). When there are algebraic constraints among coordinated variables or when coordinated variables include both fast variation components and slow variation components, each agent can only be modeled as a singular system rather than a normal one. One typical example is three-link manipulator show in [34], which can be used to clean the facade of a very large building. Another example is multiagent supporting systems in earthquake damage-preventing buildings, water-floating plants, largediameter parabolic antennae, or telescopes. As shown in [35], a MASS can only be describe by a singular multiagent system if it consists of a lot of independent blocks and each block is supported by several pillars. Xi et al. (see [35-37]) addressed consensus problems of high-order singular multiagent systems via linear matrix inequality (LMI) constrains and matrix equality constraints. Yang et al. (see [34]) studied consensus problems for singular multiagent systems with output feedback consensus protocols. Zhou et al. [21] analyzed the stability of switched linear singular systems. These study transformed singular system into two restricted equivalent subsystems: slow subsystem and quick subsystem, which is complicated. The current paper uses the Drazin inverse theory to analyze the singular multiagent systems which can easily extend the normal system results to singular system. The Drazin inverse theorem which is a kind of generalized inverse was firstly presented by Drazin (see $[38,39])$. Campbell et al. (see $[40,41]$ ) used the Drazin inverse to give the solutions of singular system. More knowledge about the Drazin inverse readers can get from [41-44].

Compared with the existing works related to consensus, the current paper is characterized with the following novel features. First the current paper focuses on swarm stability problem of high-order singular multiagent systems; our results show that consensus achievement is a specific case of swarm stability, which will be called asymptotic swarm stability. Second the main contribution of this paper is the presentation of necessary and sufficient conditions for the swarm stability of LTI singular systems with a general highorder model. The model in $[34,35]$ can be seen as special case of our results. By far, most of the linear high-order swarm system models studied by other scholars on consensus problems are more restrictive than the one in the current paper. Third some necessary and sufficient conditions based on the firstly utilization of the Drazin inverse theory to analyze the singular multiagent system are given, and a tool is presented to analyze the singular multiagent systems as well as the nonsingular systems. When index of the Drazin inverse is zero, these results (see $[18,32,35,45]$ ) in normal system are actually the current paper's corollary. The initial condition with nonadmissible bounded value is illustrated.

This paper is organised as follows. In Section 2, some basic lemmas and problem descriptions are introduced. In Section 3, the swarm stability problem and the LTI singular swarm system model are described. In Section 4, a necessary and sufficient condition for swarm stability is expounded. Section 4 provides a necessary and sufficient condition for asymptotic swarm stability. Some numerical simulations are discussed in Section 5. Finally, Section 6 concludes this paper.

\section{Preliminaries and Problem Descriptions}

\subsection{Some Lemmas and Definitions}

Definition 1 (see [33]). For any given two matrices $E, A \in$ $\mathbb{R}^{n \times n},(E, A)$ is called regular if there exists a constant scalar $\alpha \in \mathbb{C}$ such that $\operatorname{det}(\alpha E+A) \neq 0$.

Lemma 2 (see [33]). For any given two matrices $E, A \in \mathbb{R}^{n \times n}$, $(E, A)$ is regular if and only if there exist two nonsingular matrices $Q, P \in \mathbb{R}^{n \times n}$ such that

$$
Q E P=\operatorname{diag}\left\{I_{n_{1}}, N\right\}, \quad Q A P=\operatorname{diag}\left\{A_{1}, I_{n_{2}}\right\},
$$

where $n_{1}+n_{2}=n, A_{1} \in \mathbb{R}^{n_{1} \times n_{1}}, N \in \mathbb{R}^{n_{2} \times n_{2}}$ is nilpotent.

Lemma 3 (see [33]). For the regular singular system $E \dot{x}(t)=$ $A x(t)+B u(t)$ with $E, A \in \mathbb{R}^{n \times n}$ and $B \in \mathbb{R}^{n \times d}$,

(1) the system $(E, A, B)$ does not have impulse-modes called system impulse-free if and only if $\operatorname{rank}\left[\begin{array}{cc}E & 0 \\ A & E\end{array}\right]=$ $n+\operatorname{rank}(E)$ or $\operatorname{deg} \operatorname{det}(s E-A)=\operatorname{rank}(E)$,

(2) the system $(E, A, B)$ is impulse controllable if and only if $\operatorname{rank}\left[\begin{array}{ccc}E & 0 & 0 \\ A & E & B\end{array}\right]=n+\operatorname{rank}(E)$.

Definition 4 (see [40]). Let $A \in \mathbb{C}^{n \times n}$, where the index of $A$ is the smallest nonnegative integer $k$ such that $\operatorname{rank}\left(A^{k+1}\right)=$ $\operatorname{rank}\left(A^{k}\right)$ is denoted by $\operatorname{Ind}(A)$. For a square matrix $A$, the unique matrix $X$ satisfying the following relations

$$
X A X=X, \quad A X=X A, \quad A^{k+1} X=A^{k}
$$

is called the Drazin inverse of $A$ and denoted by $X=A^{D}$. Particularly, when $\operatorname{Ind}(A)=0$, the matrix $X$ is $A^{-1}$.

Some Drazin inverse lemmas will be introduced.

Lemma 5 (see [46]). If $\operatorname{Index}(A)=k, \operatorname{rank}\left(A^{k}\right)=r$ and $P$, $Q$, and $C$ are invertible, $N$ is nilpotent of index $k$, the Drazin inverse of $B$ is $B^{D}$, and the Drazin inverse of $A$ is $A^{D}$, then 
(1) $\left(Q A Q^{-1}\right)^{D}=Q A^{D} Q^{-1}$,

(2) $(A \otimes B)^{D}=A^{D} \otimes B^{D}$,

(3) if A have a Jordan canonical form $A=P\left[\begin{array}{cc}C & 0 \\ 0 & N\end{array}\right] P^{-1}$, then the Drazin inverse can be computed as

$$
A^{D}=P\left[\begin{array}{cc}
C^{-1} & 0 \\
0 & 0
\end{array}\right] P^{-1} .
$$

Lemma 6. For any given $E, A \in \mathbb{R}^{n \times n}$ and if $(E, A)$ is regular, let $\widetilde{E}=(\alpha E+A)^{-1} E$ and $\widetilde{A}=(\alpha E+A)^{-1} A$. There exist two nonsingular matrices $Q, P \in \mathbb{R}^{n \times n}$, such that

$$
\begin{aligned}
(\widetilde{E})^{D} & =P\left[\begin{array}{ll}
I_{n_{1}} & \\
& O_{n_{2}}
\end{array}\right] Q(\alpha E+A), \\
(\widetilde{E})^{D} \widetilde{A} & =P\left[\begin{array}{ll}
A_{1} & \\
& O_{n_{2}}
\end{array}\right] P^{-1}=\left(P\left[\begin{array}{ll}
I_{n_{1}} & \\
& O_{n_{2}}
\end{array}\right] Q\right) A, \\
(\widetilde{E})^{D} \widetilde{E} & =P\left[\begin{array}{ll}
I_{n_{1}} & \\
& O_{n_{2}}
\end{array}\right] P^{-1}
\end{aligned}
$$

with $A_{1} \in \mathbb{R}^{n_{1} \times n_{1}}, n_{1}+n_{2}=n, \alpha \in \mathbb{C}$ is a constant scalar, and $\mathrm{O}_{n_{2}}$ is a zero matrix.

Proof. For $(E, A)$ is regular, by Lemma 2, there exist two nonsingular matrices $Q, P \in \mathbb{R}^{n \times n}$; we can get $E=$ $Q^{-1} \operatorname{diag}\left\{I_{n_{1}}, N\right\} P^{-1}, A=Q^{-1} \operatorname{diag}\left\{A_{1}, I_{n_{2}}\right\} P^{-1}$, where $A_{1} \in$ $\mathbb{R}^{n_{1} \times n_{1}}, N \in \mathbb{R}^{n_{2} \times n_{2}}$, and $n_{1}+n_{2}=n, N$ is nilpotent matrix. We get that

$$
\begin{aligned}
\widetilde{E} & =(\alpha E+A)^{-1} E \\
& =P\left[\begin{array}{ll}
\left(\alpha I_{n_{1}}+A_{1}\right)^{-1} & \\
& \left(\alpha N+I_{n_{2}}\right)^{-1}
\end{array}\right] Q Q^{-1}\left[\begin{array}{ll}
I_{n_{1}} & \\
& N
\end{array}\right] P^{-1} \\
& =P\left[\begin{array}{ll}
\left(\alpha I_{n_{1}}+A_{1}\right)^{-1} & \left(\alpha N+I_{n_{2}}\right)^{-1} N
\end{array}\right] P^{-1}
\end{aligned}
$$

for $\left(\alpha N+I_{n_{2}}\right)^{-1} N$ is nilpotent, and with Lemma 5 we can get

$$
\begin{aligned}
(\widetilde{E})^{D} & =P\left[\begin{array}{lll}
\alpha I_{n_{1}}+A_{1} & \\
& & O_{n_{2}}
\end{array}\right] P^{-1} \\
& =P\left[\begin{array}{ll}
I_{n_{1}} & \\
& O_{n_{2}}
\end{array}\right] Q Q^{-1}\left[\begin{array}{ll}
\alpha I_{n_{1}}+A_{1} & \\
& \alpha N+I_{n_{2}}
\end{array}\right] P^{-1} \\
& =P\left[\begin{array}{ll}
I_{n_{1}} & \\
& O_{n_{2}}
\end{array}\right] Q(\alpha E+A),
\end{aligned}
$$

and the other results are easily to get.
Lemma 7. If $E$ is a nonsingular matrix in Lemma 6, suppose that $\widetilde{E}=(\alpha E+A)^{-1} E, \widetilde{A}=(\alpha E+A)^{-1} A$. Then

$$
\begin{aligned}
(\widetilde{E})^{D} & =E^{-1}(\alpha E+A), \\
(\widetilde{E})^{D} \widetilde{A} & =E^{-1} A, \\
(\widetilde{E})^{D} \widetilde{E} & =I .
\end{aligned}
$$

Lemma 8 (see [39]). For $A, B \in \mathbb{R}^{n \times n}$, regular system $A \dot{x}(t)+$ $B x(t)=f(t)$ has unique solutions for admissible conditions. Let $\alpha$ be a number such that $\widehat{A}=(\alpha A+B)^{-1} A, \widehat{B}=(\alpha A+B)^{-1} B$, $\widehat{f}(t)=(\alpha A+B)^{-1} f(t)$, and $k=\operatorname{Ind}(\widehat{A})$. Then

$$
\begin{aligned}
x(t)= & e^{-\widehat{A}^{D} \widehat{B} t} \widehat{A}^{D} \widehat{A} x(0) \\
& +\widehat{A}^{D} e^{-\widehat{A}^{D} \widehat{B} t} \int_{0}^{t} e^{\widehat{A}^{D} \widehat{B} s} \widehat{f}(s) d s \\
& +\left(I-\widehat{A}^{D} \widehat{A}\right) \sum_{n=0}^{k-1}(-1)^{n}\left(\widehat{A} \widehat{B}^{D}\right)^{n} \widehat{A}^{D} \widehat{f}^{(n)}(t) .
\end{aligned}
$$

A directed weighted graph $G$ consists of a note set $V(G)=$ $\left\{v_{1}, v_{2}, \ldots, v_{n}\right\}$ and an edge set $E(G) \subseteq\left\{\left(v_{i}, v_{j}\right): v_{i}, v_{j} \in\right.$ $V(G)\}$, an adjacency matrix of graph $G$ is defined by $W=$ $\left[w_{i j}\right] \in \mathbb{R}^{n \times n}$, and $w_{i j}$ is weight of $\left(v_{i}, v_{j}\right),\left(v_{i}, v_{j}\right) \in E(G)$ if only if $w_{i j}>0$. Moreover, it is supposed that $w_{i j}=0$ for all $i \in\{1,2, \ldots, n\}$. The set of neighbors of $v_{i}$ is denoted by $N_{i}=\left\{v_{j} \in V(G):\left(v_{i}, v_{j}\right) \in E(G)\right\}$. The Laplacian matrix of graph $G$ is defied as $L=D-W$, where $D$ is the in-degree matrix of $G$. A directed weighted graph having a spanning tree means that there exists at least one node having a directed path to all other nodes.

Lemma 9 (see [24]). Let $L \in \mathbb{R}^{n \times n}$ be the Laplacian matrix of a directed graph $G$ and $1_{n}=[1,1, \ldots, 1]^{T} \in \mathbb{R}^{n}$. Then

(1) L at least has a zero eigenvalue and $1_{n}$ is the associated eigenvector, that is, $L 1_{n}=0$,

(2) if graph $G$ has a spanning tree, then 0 is a simple eigenvalue of $L$, and all the other $n-1$ eigenvalues have positive real parts.

\subsection{Description of Singular Multiagent Continuous-Time Systems}

Assumption 10. The systems which the current paper studies are regular and impulse-free.

The regularity condition could guarantee that the system has a unique solution. The regularity condition is deemed as a kind of singular system (see [33]). If the continuous-time system has strong impulse modes, the impulse may stop the system from working or even destroy it. Readers can get the example from [47].

This section introduces our multiagent model. Suppose that the multiagent system consists of $n$ autonomous agents, labeled $i$ through $n$, and all these agents share a common state 
space $\mathbb{R}^{m}$. Let $x_{i}(t)$ denote the state of agent $i, i \in\{1,2, \ldots, n\}$, and let $x(t)=\left[x_{1}^{T}(t), x_{2}^{T}(t), \ldots, x_{n}^{T}(t)\right]^{T}$. We say that $i$ th and $j$ th agents on a network agree if and only if $x_{i}(t)=x_{j}(t)$ and that the agents on a network reached a consensus if and only if $x_{i}(t)=x_{j}(t)$ for all $i, j \in\{1,2, \ldots, n\}, i \neq j[23]$. Whenever the agents of a network are all in agreement, the common value of all agents is called group decision or consensus state.

Now we take a typical example, and we also consider the dynamics of the $n$ agents where the communication topology is represented by $G$ and each vertex corresponds to an agent. Suppose that these agents are with the following dynamics:

$$
E \dot{x}_{i}(t)=A x_{i}(t)+B u_{i}(t)
$$

and system with state feedback:

$$
E \dot{x}_{i}(t)=A x_{i}(t)+B K_{1} x_{i}(t)+B u_{i}(t)
$$

where $E \in \mathbb{R}^{m \times m}, A \in \mathbb{R}^{m \times m}, B \in \mathbb{R}^{m \times d}$, and $K_{1} \in \mathbb{R}^{d \times m}$. As we can see when system with state feedback is actually special case of system (9) for $E \dot{x}_{i}(t)=\left(A+B K_{1}\right) x_{i}(t)+B u_{i}(t)$ with $\operatorname{rank}(E)=c \leq m$ and $u_{i}(t)$ is the consensus protocol which is designed according to the information available to agent $i$, the following consensus protocol is applied:

$$
u_{i}(t)=K_{2} \sum_{j \in n_{i}} w_{i j}\left(x_{j}(t)-x_{i}(t)\right)
$$

where $i \in\{1,2, \ldots, n\}, K_{1}, K_{2} \in \mathbb{R}^{d \times m}$. Let $x(t)=\left[x_{1}^{T}(t)\right.$, $\left.x_{2}^{T}(t), \ldots, x_{n}^{T}(t)\right]^{T}$, and let $L$ be the Laplacian matrix of graph $G$. Then the dynamics of a singular swarm system with $n$ agents can be described by

$$
\left(I_{n} \otimes E\right) \dot{x}(t)=H x(t)=\left(I_{n} \otimes A-L \otimes B K_{2}\right) x(t) .
$$

For the Laplacian matrix $L \in \mathbb{R}^{n \times n}$ of the interaction topology $G$, there exists a nonsingular matrix $U \in \mathbb{C}^{n \times n}$ such that $U^{-1} L U=J_{L}$ where $J_{L}$ is a Jordan canonical form of $L$. Let $\bar{x}(t)=\left(\bar{x}_{1}^{H}(t), \bar{x}_{2}^{H}(t), \ldots, \bar{x}_{n}^{H}(t)\right)^{H}=\left(U^{-1} \otimes I_{n}\right) x(t)$, where $I_{n}$ is identity matrix. Let $H_{\lambda_{i}}=A-\lambda_{i} B K_{2}, i \in\{1,2, \ldots, n\}$. Then by Lemma 20 system, (12) can be transformed in to

$$
\begin{aligned}
\left(I_{n} \otimes E\right) \dot{\tilde{x}}(t) & =\widehat{H} \tilde{x}(t)=\left(I_{n} \otimes A-J_{L} \otimes B K_{2}\right) \tilde{x}(t) \\
& =\left[\begin{array}{ccccc}
H_{\lambda_{1}} & * & & & \\
& H_{\lambda_{2}} & * & & \\
& & \ddots & \ddots & \\
& & & H_{\lambda_{n-1}} & * \\
& & & & H_{\lambda_{n}}
\end{array}\right] \tilde{x}(t),
\end{aligned}
$$

where $*$ denotes a block in $\mathbb{R}^{m \times m}$ that may either be $-B K_{2}$ or 0 .

Definition 11 (out stability). For an LTI singular system

$$
\begin{aligned}
& E \dot{x}=A x, \\
& y=C x,
\end{aligned}
$$

suppose that, for $\forall \varepsilon>0, \exists \delta(\varepsilon)>0$ s.t. $\|y(t)\|<\varepsilon(t>0)$ as $\|y(0)\|<\delta(\varepsilon)$. Then the system is said to be output stable with respect to $y$.
Define the auxiliary vectors $\eta_{i}(t)=\sum_{j \in n_{i}} w_{i j}\left(x_{i}(t)-\right.$ $\left.x_{j}(t)\right)(i \in\{1,2, \ldots, n\})$ and the stack of them as $\eta(t)=$ $\left[\eta_{1}^{T}(t), \eta_{2}^{T}(t), \ldots, \eta_{n}^{T}(t)\right]^{T}$. It follows that

$$
\eta=\left(L \otimes I_{m}\right) x(t)
$$

As a result of $(15)$ and $\bar{x}(t)=\left(U^{-1} \otimes I_{n}\right) x(t)$,

$$
\begin{aligned}
\left(U^{-1} \otimes I_{n}\right) \eta & =\left(U^{-1} \otimes I_{n}\right)\left(L \otimes I_{m}\right) x(t)=\left(U^{-1} L \otimes I_{m}\right) x(t) \\
& =\left(U^{-1} L \otimes I_{m}\right)\left(U \otimes I_{n}\right) \bar{x}(t)=\left(J \otimes I_{n}\right) \bar{x}(t) .
\end{aligned}
$$

For the system (9), we said that the system is stable like the normal linear system case; when studying stability of singular linear systems, we need only to consider the following homogeneous equation:

$$
E \dot{x}_{i}(t)=A x_{i}(t),
$$

where $E \in \mathbb{R}^{m \times m}, A \in \mathbb{R}^{m \times m}, B \in \mathbb{R}^{m \times d}$, with $\operatorname{rank}(E)=c \leq$ $m$, and $x_{i}(0)$ is the admissible initial condition.

System (17) satisfies the regularity condition, and there exists a scalar $\alpha$ such that $\operatorname{det}(\alpha E+A) \neq 0$. The system (17) can be transformed into the equivalent system

$$
\widetilde{E} \dot{x}(t)=\widetilde{A} x(t),
$$

where $\widetilde{E}=(\alpha E+A)^{-1} E, \widetilde{A}=(\alpha E+A)^{-1} A$. The regularity condition assures that the system (17) has solution (see [33])

$$
x(t)=e^{\widetilde{E}^{D} \widetilde{A} t} \widetilde{E}^{D} \widetilde{E} x(0)
$$

and $x(0)$ is admissible initial condition.

By Lemmas 2 and 6, there exist two nonsingular matrices $\mathrm{Q}, P \in \mathbb{R}^{m n \times m n}$; we can get

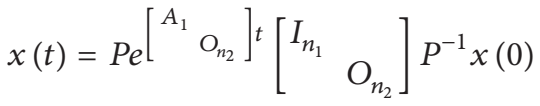

$$
\begin{aligned}
& =P\left[\begin{array}{ll}
e^{A_{1} t} & \\
& O_{n_{2}}
\end{array}\right] P^{-1} x(0) \text {, }
\end{aligned}
$$

where $A_{1} \in \mathbb{R}^{n_{1} \times n_{1}}, n_{1}+n_{2}=n m$.

Remark 12. From the structure of the solution we can see that $\widetilde{E}^{D} \widetilde{A}$ has two-part eigenvalues, eigenvalues of part $A_{1}$ and eigenvalues of part $O_{n_{2}}$. Eigenvalues of part $A_{1}$ are effective eigenvalues. In this current paper slow subsystem eigenvalues of $(E, A)$ represent eigenvalues of part $A_{1}$. Let $(E, A)$ represent $\widetilde{E}^{D} \widetilde{A}$.

Definition 13 (see [48]). Consider the system (17) or the matrix pair $(E, A)$ where $E \in \mathbb{R}^{m \times m}, A \in \mathbb{R}^{m \times m}$.

(1) A pole of the system, or a finite eigenvalue of the matrix pair $(E, A)$, is called stable if it has negative real part, is called critically stable if it has zero real part, and is called unstable if it has positive real part. 
(2) The matrix pair $(E, A)$ is called stable if it has only stable finite eigenvalues, is called critically stable if it has critical finite eigenvalues but not unstable ones, and is called unstable if it has unstable finite eigenvalues.

Remark 14. For a clear expression, the LTI singular systems is said to be stable if 0 is a stable equilibrium point of this system.

Definition 15 (swarm stability). Consider LTI singular multiagent systems with $n$ agents and $x_{1}, x_{2}, \ldots, x_{n} \in \mathbb{R}^{m}$ as the agent states. The system is said to be swarm stable if for $\forall \varepsilon>0, \exists \delta(\varepsilon)>0$ s.t. $\left\|x_{i}(t)-x_{j}(t)\right\|<\varepsilon(t>0)$ as $\left\|x_{i}(t)-x_{j}(t)\right\|<\delta(\varepsilon)(\forall i, j \in\{1,2, \ldots, n\})$.

Definition 16 (asymptotically swarm stability). Consider that LTI singular multiagent systems achieve full state consensus, that is. for $\forall \varepsilon>0, \exists T>0$ s.t. when $t>T, \| x_{i}(t)-$ $x_{j}(t) \|<\varepsilon(\forall i, j \in\{1,2, \ldots, n\})$. Then it is said to be asymptotically swarm stable.

The swarm stability discussed in this paper is LTI singular systems, and the definition of swarm stability and asymptotic swarm stability seem similar to [18]; however, they are actually different. The current paper focuses on the more general cases which is LTI singular systems. As well known for the system (10), when $\operatorname{rank}(E)=m$ the normal systems are actually spacial cases of the system (10).

Besides consensus achievement, some phenomena investigated by the researchers, for example, flocking and formation keeping, also require a system to be swarm stable. A bird flock, vehicle platoon, or robot crew may navigate to anywhere, but the distances among its members should not go unbounded. The states of agents in a swarm stable system might still oscillate or even diverge. Swarm stability is a kind of nonequilibrium stability [18].

\section{Swarm Stability}

The major purpose of this section is to present a necessary and sufficient condition for the swarm stability of high-order LTI singular swarm systems. For this end, several preparations are needed.

Lemma 17. For multiagent systems (9), if it is asymptotically swarm stable, then as $t \rightarrow \infty$, the trajectory of each agent satisfies $E \dot{\xi}-A \xi \rightarrow 0$.

Proof. Without loss of generality, consider an agent $i$. The difference between $E \dot{x}_{i}$ and $A x_{i}$ is $E \dot{x}_{i}-A x_{i}=$ $B K_{2} \sum_{j \in N_{i}} w_{i j}\left(x_{j}-x_{i}\right)$. Since the system is asymptotically swarm stable, $\lim _{t \rightarrow \infty}\left(x_{j}-x_{i}\right)=0(j \in\{1,2, \ldots, n\})$ and $\lim _{t \rightarrow \infty} B K_{2} \sum_{j \in N_{i}} w_{i j}\left(x_{j}-x_{i}\right)=0$. Thus, as $t \rightarrow \infty$, the difference $\left(E \dot{x}_{i}-A x_{i}\right) \rightarrow 0$.

Lemma 17 naturally leads to the following two corollaries.

Corollary 18. For multiagent System (9), if each slow subsystem eigenvalue of $(E, A)$ has negative real part, then the system is asymptotically swarm stable if and only if it is asymptotically stable and the limit of all agents state that is the consensus state, is zero.

Proof. For the LTI singular systems (9), like the normal linear system case, when studying stability of singular linear systems, we need only to consider the following homogeneous equation:

$$
E \dot{x}_{i}=A x_{i}
$$

for $i \in\{1,2, \ldots, n\}$. The regularity condition assures that the system (9), by the Lemma 8 we can get

$$
x_{i}(t)=e^{\widetilde{E}^{D} \widetilde{A}} \widetilde{E}^{D} \widetilde{E} x_{i}(0)
$$

with $\widetilde{E}=(\alpha E+A)^{-1} E$ and $\widetilde{A}=(\alpha E+A)^{-1} A, x_{i}(0)$ is the admissible initial condition. As we can see that each slow subsystem eigenvalue of $(E, A)$ has negative real part, the limit $\lim _{t \rightarrow \infty} x_{i}(t)=\lim _{t \rightarrow \infty} e^{\widetilde{E}^{D} \widetilde{A}} \widetilde{E}^{D} \widetilde{E} x(0)=0$, so the consensus state, is zero and it is asymptotically stable with Lemma 17 from which we can get the results.

Corollary 19. For singular multi agent system (9), the system is asymptotically swarm stable and the consensus state is not zero. Then not each slow subsystem eigenvalues of $(E, A)$ has negative real part.

From the proof of Corollary 18, we can easily get the results.

Lemma 20. For LTI singular multi agent system (9), if $(E, A)$ is stable, then swarm stability of the system is equivalent to stable.

Proof. Suppose that the system is swarm stable. If $\left\|x_{i}(0)\right\|<$ $\delta / 2(i \in\{1,2, \ldots, n\})$, then $\left\|x_{i}(0)-x_{j}(0)\right\|<\delta(i, j \in$ $\{1,2, \ldots, n\})$. Owning to the swarm stability, for $\forall \varepsilon>0, \exists \delta(\varepsilon)$ s.t. $\left\|x_{i}(t)-x_{j}(t)\right\|<\varepsilon(j \in\{1,2, \ldots, n\})$ as $\left\|x_{i}(0)\right\|<\delta / 2$ $(i \in\{1,2, \ldots, n\}) . \varepsilon$ can be arbitrarily small if with proper $\delta$. Let $\varepsilon \rightarrow 0$. According to the similar idea in the proof of Lemma 17 , the motion of each agent can satisfy $E \dot{\xi}-A \xi \rightarrow 0$. Assume that the swarm system is unstable. Then the agent states may diverge since the system is LTI. This contradicts the fact that $(E, A)$ is stable. Thus, the multi agent system must be stable.

Suppose that the system is stable. Assume that the system is swarm unstable. There may exist a pair of agents $i$ and $j$ with $\left(x_{i}-x_{j}\right) \rightarrow \infty$ as $t \rightarrow \infty$. This implicates that at least the state of one agent goes unbounded. The assumption contradicts the fact that the system is stable. Thus, the system is swarm stable.

Lemma 21. If the graph $G$ includes a spanning tree, then a necessary and sufficient condition for the swarm stability of the singular system is that it is output stable with respect to $\eta$.

Proof. Owing to the definition of $\eta$ the system is output stable with respect to $\eta$ if it is swarm stable. According to Lemma 20, 
if the graph $G$ includes a spanning tree, then there exists certain nonsingular matrix $P \in \mathbb{R}^{n \times n}$ s.t.

$$
P L=\Gamma=\left[\begin{array}{ccccc}
1 & -1 & 0 & & \\
0 & 1 & \ddots & \ddots & \\
& \ddots & \ddots & -1 & 0 \\
& & 0 & 1 & -1 \\
* & * & \cdots & \cdots & *
\end{array}\right]
$$

where the first $n-1$ rows in $\Gamma$ form a basis for the row space of $L$ and $*$ can be any value. Thus, (15) can be transformed into $\left(P \otimes I_{d}\right) \eta=\left(\Gamma \otimes I_{d}\right) x$. All the vectors $x_{1}-x_{2}, x_{2}-$ $x_{3}, \ldots, x_{n-1}-x_{n}$ are dependent on $\eta$. The value of any $\left\|x_{i}-x_{j}\right\|$ $(i, j \in\{1,2, \ldots, n\})$ can be arbitrarily small if $\eta$ is sufficiently close to zero. Therefore, the system is swarm stable if it is output stable with respect to $\eta$.

This section will establish some necessary and sufficient conditions guaranteeing that the system has a consensus property.

Theorem 22. For swarm system (9) with $\lambda_{1}=0, \lambda_{2}, \ldots, \lambda_{n} \in$ $\mathbb{C}$ as the eigenvalues of $L(G)$, if $(E, A)$ is unstable, the system is swarm stable if and only if both (1) and (2) below are true:

(1) G includes a spanning tree;

(2) all the (E, $\left.A-\lambda_{i} B K_{2}\right),\left(\lambda_{i} \neq 0\right)$, are stable. Besides, if, for some $i,\left(E, A-\lambda_{i} B K_{2}\right)$ is critically stable and $L$ is not diagonalisable, each submatrix in $\left(I_{n} \otimes E, I_{n} \otimes A-\right.$ $J \otimes B K_{2}$ ) corresponding to a Jordan block of $\lambda_{i}$ which has the form

$$
\begin{gathered}
\left(\left[\begin{array}{llll}
E & & & \\
& E & & \\
& & \ddots & \\
& & & E
\end{array}\right],\right. \\
\left.\left[\begin{array}{cccc}
A-\lambda_{i} B K_{2} & -B K_{2} & 0 & \\
0 & A-\lambda_{i} B K_{2} & \ddots & 0 \\
\vdots & & \ddots & -B K_{2} \\
0 & \ldots & 0 & A-\lambda_{i} B K_{2}
\end{array}\right]\right)
\end{gathered}
$$

is stable.

If $(E, A)$ is stable, then the system is swarm stable if and only if condition (2) is true.

Proof. (E, A) Is Unstable. Assume that $G$ does not include a spanning tree while the system is still swarm stable. $\exists \delta(\varepsilon)>0$ s.t. $\left\|x_{i}(t)-x_{j}(t)\right\|<\varepsilon$ if $\left\|x_{i}(0)-x_{j}(0)\right\|<\delta(\forall i, j \in$ $\{1,2, \ldots, n\})$. $G$ must contain $k \geq 2$ different subgraphs $\widehat{G}_{1}, \widehat{G}_{2}, \ldots, \widehat{G}_{k}$ each receiving no information. According to the basic idea in the proof of Lemmas 17 and 20, $\varepsilon$ can be arbitrarily small if $\delta$ is small enough. Let $\varepsilon \rightarrow 0$. Then the motion of each agent associated with $\widehat{G}_{1}$ can satisfy $E \dot{\xi}_{1}-$ $A \xi_{1} \rightarrow 0$, whereas the motion of each agent associated with $\widehat{G}_{2}$ can satisfy $E \dot{\xi}_{2}-A \xi_{2} \rightarrow 0$. As $\xi_{1}$ and $\xi_{2}$ are independent, $E \dot{\xi}_{1}-E \dot{\xi}_{2}-A\left(\xi_{1}-\xi_{2}\right) \rightarrow 0$. Since $(E, A)$ is unstable and the system is LTI, there exist $\xi_{1}(0) \neq \xi_{2}(0)$ s.t. $\xi_{1}(t)-\xi_{2}(t) \rightarrow \infty$ $(t \rightarrow \infty)$. This contradicts the fact that the system is swarm stable. Thus, Condition (1) is necessary.

Owing to Lemma 21, when condition (1) is true, swarm stability is equivalent to that the system is output stable with respect to $\eta$. Notice that $\lambda_{2}, \ldots, \lambda_{n}$ are all nonzero. According to the linear equation (16) and the upper triangular structure of $J$, the output stability with respect to is equivalent to the output stability with respect to $\widetilde{x}_{2}, \widetilde{x}_{3}, \ldots, \widetilde{x}_{n}$. Evidently, one can infer that condition (1) is a necessary and sufficient condition for such an output stability by considering the structure of (13).

(E, A) Is Stable. According to Lemma 20, the swarm stability of the system is equivalent to the asymptotically sta-bility of (10). One can draw the conclusion that (10) is stable if and only if Condition (2) is true by considering the structure of (13).

Remark 23. For system (9), if $\operatorname{Index}(E)=0$, the results in [18] is a special case of the Theorem 22.

Corollary 24. For swarm system (10) with $\lambda_{1}=0, \lambda_{2}, \ldots$, $\lambda_{n} \in \mathbb{C}$ as the eigenvalues of $L(G)$, if $\left(E, A+B K_{1}\right)$ is unstable, the system is swarm stable if and only if both (1) and (2) below are true:

(1) G includes a spanning tree;

(2) all the $\left(E, A+B K_{1}-\lambda_{i} B K_{2}\right), \lambda_{i} \neq 0$, are stable. Besides, iffor some $i,\left(E, A+B K_{1}-\lambda_{i} B K_{2}\right)$ is critically stable and $L$ is not diagonalisable, each submatrix in $\left(I_{n} \otimes E, I_{n} \otimes\right.$ $\left.\left(A+B K_{1}\right)-J \otimes B K_{2}\right)$ corresponding to a Jordan block of $\lambda_{i}$ which has the form

$$
\begin{gathered}
\left(\begin{array}{llll}
E & & & \\
& E & & \\
& & \ddots & \\
& & E
\end{array}\right], \\
\\
\left.\left[\begin{array}{cccc}
A+B K_{1}-\lambda_{i} B K_{2} & -B K_{2} & 0 & \\
0 & A+B K_{1}-\lambda_{i} B K_{2} & \ddots & 0 \\
\vdots & & \ddots & -B K_{2} \\
0 & \ldots & 0 & A+B K_{1}-\lambda_{i} B K_{2}
\end{array}\right]\right)
\end{gathered}
$$

is stable.

If $\left(E, A+B K_{1}\right)$ is stable, then the system is swarm stable if and only if condition (2) is true.

The proof is the same as that Theorem 22.

\section{Asymptotic Swarm Stability}

Theorem 25. For swarm system (9) with $\lambda_{1}=0, \lambda_{2}, \ldots, \lambda_{n} \in$ $\mathbb{C}$ as the eigenvalues of the Laplacian matrix $L(G)$, if not each slow subsystem eigenvalues of $(E, A)$ has negative real part, the system is asymptotically swarm stable if and only if both (1) and (2) below are true:

(1) the graph topology $G$ includes a spanning tree.

(2) each slow subsystem eigenvalue of $\left(E, A-\lambda_{i} B K_{2}\right)$ $\left(\lambda_{i} \neq 0\right)$ has negative real part. 
If each slow subsystem eigenvalue of $(E, A)$ has negative real part, then the system is asymptotically swarm stable if and only if condition (2) is true.

Proof. Part 1. Not each slow subsystem eigenvalue of $(E, A)$ has negative real part. According to the same approach in the proof of Theorem 22, it is easy to prove that Condition (1) is necessary. With Condition (1), according to Lemma 20, any vector in the null space of $L \otimes I_{d}$ is of the form $\phi \otimes \xi$ where $\phi=[1,1, \ldots, 1]^{T} \in \mathbb{R}^{m}$ and $\xi \in \mathbb{R}^{m}$. Therefore $x_{1}=$ $x_{2}=\cdots=x_{n}$ if and only if $\eta=0$. According to Lemma 20, $\lambda_{2}, \ldots, \lambda_{n}$ are all nonzero. From the linear equation (16), one knows that $\eta=0$ if and only if $\tilde{x}_{2}=\cdots=\tilde{x}_{n}=0$ with considering the triangular structure of $J$. According to (13), $I_{n} \otimes A-J \otimes B K_{2}$ is a strictly upper triangular block matrix and the stability of (10) is determined by the eigenvalues of ( $E, A-$ $\left.\lambda_{i} B K_{2}\right)$. Meanwhile, the output stability of the system (9) respect to $\widetilde{x}_{2}, \widetilde{x}_{3}, \ldots, \widetilde{x}_{n}$ is independent of $\widetilde{x}_{1}$. Consequently, $\tilde{x}_{2}, \widetilde{x}_{3}, \ldots, \widetilde{x}_{n} \rightarrow 0(t \rightarrow \infty)$ if and only if each slow subsystem eigenvalue of $\left(E, A-\lambda_{2} B K_{2}\right), \ldots,\left(E, A-\lambda_{n} B K_{2}\right)$ has negative real part.

Part 2. Each slow subsystem eigenvalue of $(E, A)$ has negative real part. When each slow subsystem eigenvalue of $(E, A)$ has negative real part, according to Corollary 18, the multiagent system's asymptotic swarm stability is equivalent to its asymptotic stability. The stability of system (9) is equivalent to that of (10). By observing the structure of $I_{n} \otimes A-J \otimes$ $B K_{2}$ in (13), it is easy to infer that (10) is asymptotically stable if and only if condition (2) is true.

Remark 26. For system (9), if $\operatorname{Index}(E)=0$, the results in $[18,34]$ is a special case of Theorem 25 .

Corollary 27. For swarm system (10) with $\lambda_{1}=0, \lambda_{2}, \ldots$, $\lambda_{n} \in \mathbb{C}$ as the eigenvalues of the Laplacian matrix $L(G)$, if not each slow subsystem eigenvalue of $\left(E, A+B K_{1}\right)$ has negative real part, the system is asymptotically swarm stable if and only if both (1) and (2) below are true:

(1) the graph topology $G$ includes a spanning tree.

(2) each slow subsystem eigenvalue of $\left(E, A+B K_{1}-\lambda_{i} B K_{2}\right)$ $\left(\lambda_{i} \neq 0\right)$ has negative real part.

If each slow subsystem eigenvalue of $\left(E, A+B K_{1}\right)$ has negative real part, then the system is asymptotically swarm stable if and only if condition (2) is true.

Remark 28. For system (10), the result in $[18,32,35]$ is a special case of the Corollary 27.

\section{Simulation}

In this section, numerical instances will be exhibited to illustrate the theoretical results in the previous section, three graphs $G_{1}, G_{2}$, and $G_{3}$.

5.1. First Example. A network with three nodes and four edges, whose topology is shown in Figure 1, the eigenvalues

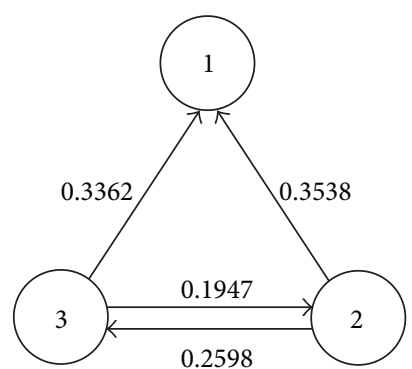

FIGURE 1: Direction interaction topology graph $G_{1}$.
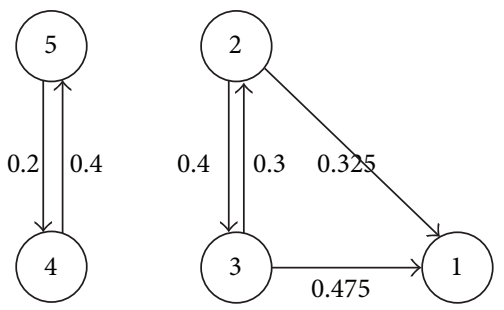

FIGURE 2: Direction interaction topology graph $G_{2}$.

of the Laplacian matrices for the two graphs are $\lambda\left(G_{1}\right)=$ $\{0,0.4545,0.69\}$ and

$$
\begin{gathered}
E=\left[\begin{array}{ccc}
0 & 1 & 0 \\
-5 & 2 & 0 \\
0 & 1 & 0
\end{array}\right], \\
A=\left[\begin{array}{ccc}
7.4197 & 0.152 & 0 \\
5.5994 & 3.4841 & 0 \\
1 & 2 & 1
\end{array}\right], \\
B K_{2}=\left[\begin{array}{lll}
1 & 4 & 0 \\
0 & 7 & 0 \\
0 & 0 & 0
\end{array}\right] .
\end{gathered}
$$

In this example, $(E, A)$ is unstable with slow subsystem eigenvalues $1 \pm 2 i, \operatorname{rank}(E)=2<3$ the multiagent system is singular multiagent system, whereas the slow subsystem eigenvalues of $\left(E, A+\lambda_{i} B K_{2}\right)(i=2,3)$ are $-0.518 \pm 0.917 i$ and $\pm 1.512 i$. According to Theorems 22 and 25 , the multiagent system is swarm stable but not asymptotically swarm stable.

Figure 4 shows the three trajectories of the agents with nonadmissible initial condition in the three dimension space. The three agents diverge, while they neither escape from each other nor achieve consensus. For clearer observation, Figure 5 shows the three trajectories representing the relative motion, that is, $x_{1}-x_{2}, x_{2}-x_{3}$ and $x_{1}-x_{3}$. It is clear that the relative motions are Lyapunov stable. The dots • represent the nonadmissible initial condition position which we set, and the dots $*$ is the admissible initial condition which the system actually starts. Figure 4 indicates that the nonadmissible initial bounded has no effect on swarm stable if the multiagent can accept the nonadmissible initial bounded value. 


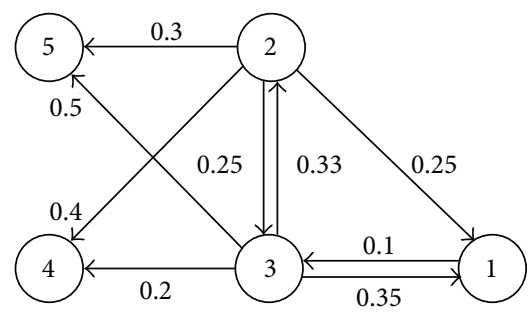

FIGURE 3: Direction interaction topology graph $G_{3}$.

5.2. Second Example. For a network with five nodes and six edges, whose topology is shown in Figure 2, the eigenvalues of the Laplacian matrices for the two graphs are $\lambda\left(G_{2}\right)=$ $\{0,0,0.6,0.7,0.8\}$ and

$$
\begin{gathered}
E=\left[\begin{array}{ccc}
0 & 1 & 0 \\
-5 & 2 & 0 \\
0 & 0 & 0
\end{array}\right], \\
A=\left[\begin{array}{ccc}
7.4197 & 0.152 & 0 \\
5.5994 & 3.4841 & 0 \\
1 & 0 & 1
\end{array}\right], \\
B K_{2}=\left[\begin{array}{lll}
1 & 4 & 0 \\
0 & 7 & 0 \\
0 & 0 & 0
\end{array}\right] .
\end{gathered}
$$

In this example, $(E, A)$ is unstable with slow subsystem eigenvalues $1 \pm 2 i, \operatorname{rank}(E)=2<3$ the multiagent system is singular swarm systems, whereas the slow subsystem eigenvalues of $\left(E, A+\lambda_{i} B K_{2}\right)\left(\lambda_{i} \neq 0\right)$ are $-0.32 \pm 1.1994 i,-0.54 \pm 0.8780 i$, $-0.76 \pm 0.1857 i$, Although the the slow subsystem eigenvalues of $\left(E, A+\lambda_{i} B K_{2}\right)\left(\lambda_{i} \neq 0\right)$ are all with negative real parts, the system is swarm unstable according to Theorem 22 for $(E, A)$ is unstable and $G_{2}$ includes no spanning tree. For $G_{2}$ has two subgraph, each subgraph has a spanning tree, so they depart two groups. $*$ is the admissible initial bounded value position (see Figure 6).

5.3. Third Example. For a network with five nodes and nine edges, whose topology is shown in Figure 3, the eigenvalues of the Laplacian matrices for the two graphs are $\lambda\left(G_{3}\right)=$ $\{0,0.7,0.58,0.6,0.8\}$ and

$$
\begin{gathered}
E=\left[\begin{array}{ccc}
0 & 1 & 0 \\
-5 & 2 & 0 \\
0 & 0 & 0
\end{array}\right], \\
A=\left[\begin{array}{ccc}
7.4197 & 0.152 & 0 \\
5.5994 & 3.4841 & 0 \\
1 & 0 & 1
\end{array}\right], \\
B K_{2}=\left[\begin{array}{lll}
1 & 4 & 0 \\
0 & 7 & 0 \\
0 & 0 & 0
\end{array}\right] .
\end{gathered}
$$

In this example, $(E, A)$ is unstable with slow subsystem eigenvalues $1 \pm 2 i, \operatorname{rank}(E)=2<3$ the multiagent system is sin-

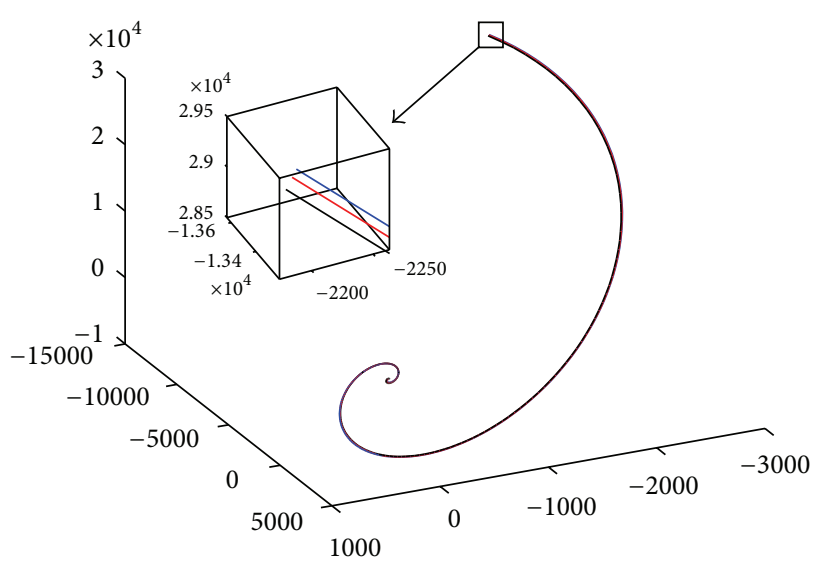

FIGURE 4: Trajectories of three agents in the first instance with nonadmissible bounded initial $t \in\left[\begin{array}{ll}0 & 7\end{array}\right]$.

gular swarm systems, whereas the slow subsystem eigenvalues of $\left(E, A+\lambda_{i} B K_{2}\right)\left(\lambda_{i} \neq 0\right)$ are $-0.276 \pm 1.2505 i,-0.54 \pm$ $0.8780 i,-0.32 \pm 0.1194 i,-0.76 \pm 0.1857 i$ although the slow subsystem eigenvalue of $(E, A)\left(\lambda_{i} \neq 0\right)$ is unstable and the system matches the condition of Theorem 25 , and it is asymptotically swarm stable. Figure 7 shows the five trajectories of the agents in the three-dimensional space. For a clear observation Figure 8 shows the four trajectories representing the relative motions that is $x_{1}-x_{2}, x_{2}-x_{3}, x_{3}-x_{4}, x_{4}-x_{5}$. It clear that the relative motions are asymptotically swarm stable.

5.4. Forth Example. For a network with five nodes and nine edges, whose topology is shown in Figure 3, the eigenvalues of the Laplacian matrices for the two graphs are $\lambda\left(G_{3}\right)=$ $\{0,0.7,0.58,0.6,0.8\}$ and

$$
\begin{gathered}
E=\left[\begin{array}{ccc}
0 & 1 & 0 \\
-5 & 2 & 0 \\
0 & 0 & 0
\end{array}\right], \\
A=\left[\begin{array}{ccc}
7.4197 & 0.152 & 0 \\
10.5994 & 1.4841 & 0 \\
1 & 0 & 1
\end{array}\right], \\
B K_{2}=\left[\begin{array}{lll}
1 & 4 & 0 \\
0 & 7 & 0 \\
0 & 0 & 0
\end{array}\right] .
\end{gathered}
$$

In this example, $(E, A)$ is critically stable with slow subsystem eigenvalues $\pm 2 i, \operatorname{rank}(E)=2<3$ the multiagent system is singular swarm systems, whereas the slow subsystem eigenvalues of $\left(E, A+\lambda_{i} B K_{2}\right)\left(\lambda_{i} \neq 0\right)$ are $-1.54 \pm 0.878 i$, $-1.276 \pm 1.2505 i,-1.32 \pm 1.994 i,-1.76 \pm 0.1857 i$ although the the slow subsystem eigenvalues of $(E, A)\left(\lambda_{i} \neq 0\right)$ is critically stable and the system matches the conditions of Theorem 25. It is asymptotically swarm stable and they achieve consensus with the consensus function is time varying. Figure 9 shows the five trajectories of the agents in the three-dimensional space. $*$ is the starting positions of agents. 


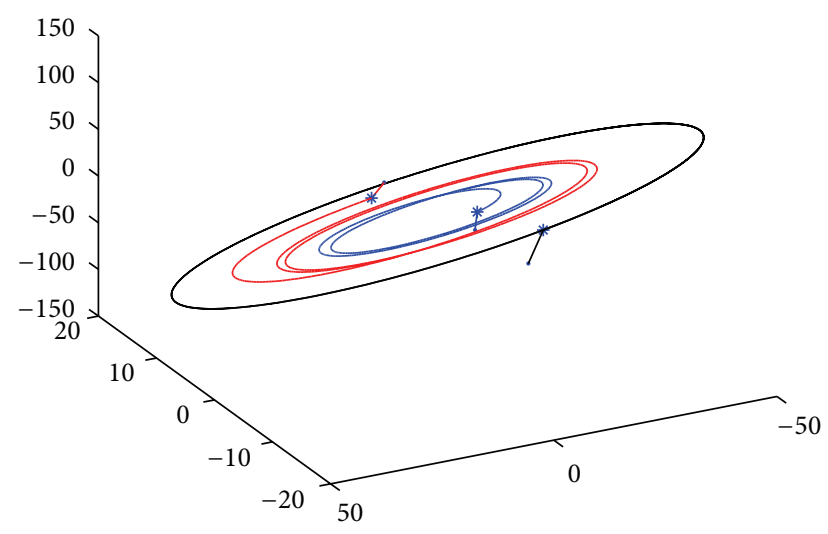

FIGURE 5: Relative motions in the first instance with non-admissible bounded initial $t \in\left[\begin{array}{ll}0 & 7\end{array}\right]$.

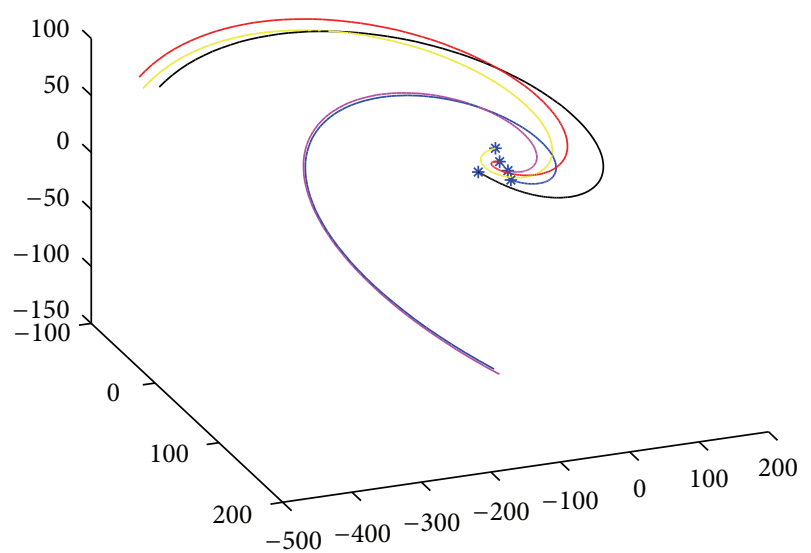

FIGURE 6: Trajectories of five agents in the second instance with admissible bounded initial $t \in\left[\begin{array}{ll}0 & 3.2\end{array}\right]$.

5.5. Fifth Example. For a network with three nodes and four edges, whose topology is shown in Figure 1, the eigenvalues of the Laplacian matrices for the two graphs are $\lambda\left(G_{1}\right)=$ $\{0,0.4545,0.69\}$ and

$$
\begin{aligned}
E & =\left[\begin{array}{cccccc}
0 & 1 & 8 & 2 & 1 & 0 \\
-5 & 2 & 6 & 1 & 1 & -1 \\
-2 & -4 & -8 & 8 & 0 & 0 \\
-1 & -2 & -4 & -2 & 2 & 0 \\
0 & 0 & 0 & 0 & 0 & 0 \\
0 & 0 & 0 & 0 & 0 & 0
\end{array}\right] \\
B K_{2} & =\left[\begin{array}{cccccc}
-1 & -4 & -5 & 4 & 5 & -6 \\
-20 & 7 & 4 & 8 & 0 & -5 \\
-2 & -2 & 8 & -2 & 0 & 0 \\
-8 & -1 & -5 & 2 & -2 & 0 \\
0 & 0 & 0 & 0 & 0 & 0 \\
0 & 0 & 0 & 0 & 0 & 0
\end{array}\right]
\end{aligned}
$$

A

$$
=\left[\begin{array}{cccccc}
1.2817 & -7.6558 & -19.2807 & -1.3580 & -2.1439 & -7.6522 \\
3.1890 & -8.7816 & -54.2009 & -45.3840 & 19.6020 & -4.2240 \\
-11.1773 & 0.6922 & -9.5160 & -34.5783 & 15.7559 & 1.6546 \\
-0.0788 & 1.0435 & 2.3770 & -13.6672 & 1.4261 & -1.7988 \\
2 & 1 & -2 & 4 & 4 & 2 \\
2 & 6 & 8 & 8 & 5 & 5
\end{array}\right] .
$$

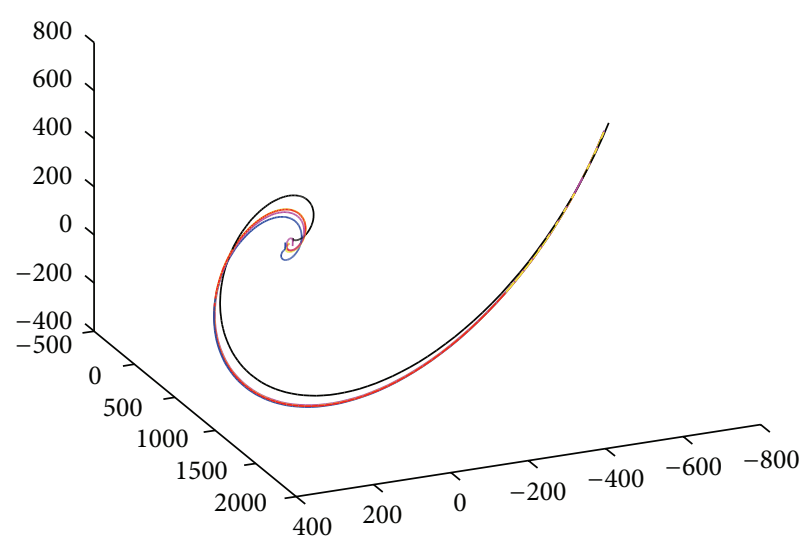

FIGURE 7: Trajectories of five agents in the third instance with admissible bounded initial $t \in\left[\begin{array}{ll}0 & 5\end{array}\right]$.

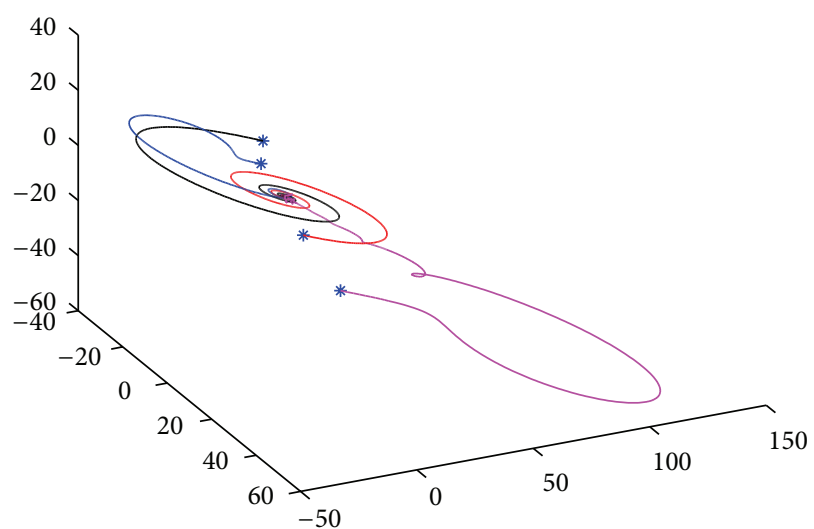

FIGURE 8: Relative motions in the third instance with admissible bounded initial $t \in\left[\begin{array}{ll}0 & 5\end{array}\right]$.

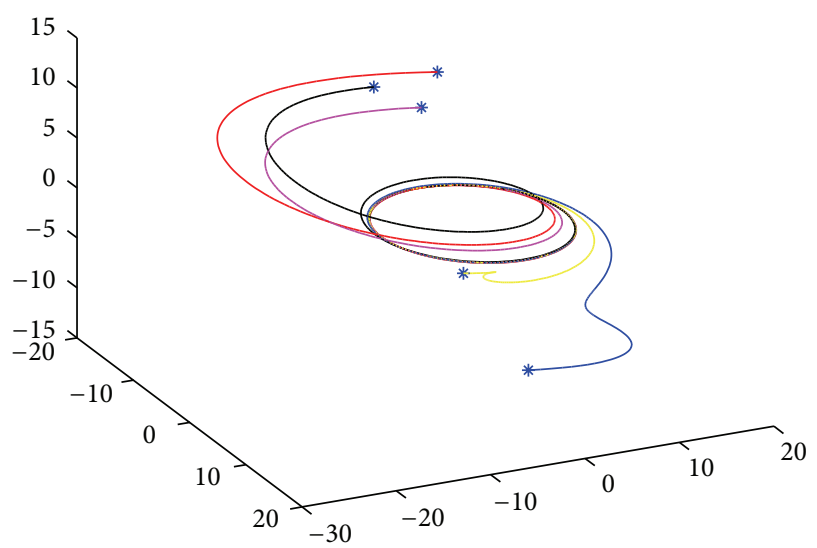

FIGURE 9: Trajectories of five agents in the forth instance with admissible bounded initial $t \in\left[\begin{array}{ll}0 & 7\end{array}\right]$.

In this example, $(E, A)$ is critically stable with slow subsystem eigenvalues $\pm 1.5773 i, \pm 3.2057 i, \operatorname{rank}(E)=4<6$ the multiagent system is singular swarm systems, whereas the slow subsystem eigenvalue of $\left(E, A+\lambda_{i} B K_{2}\right)\left(\lambda_{i} \neq 0\right)$ are $-1.0998 \pm$ $1.0758 i,-0.5158 \pm 4.1064 i,-1.7073 \pm 0.6370 i,-0.7455 \pm$ $4.4668 i$, the slow subsystem eigenvalues of $(E, A)\left(\lambda_{i} \neq 0\right)$ is 
$\left[x_{i 4}(t), x_{i 5}(t), x_{i 6}(t)\right], i \in 1,2,3$

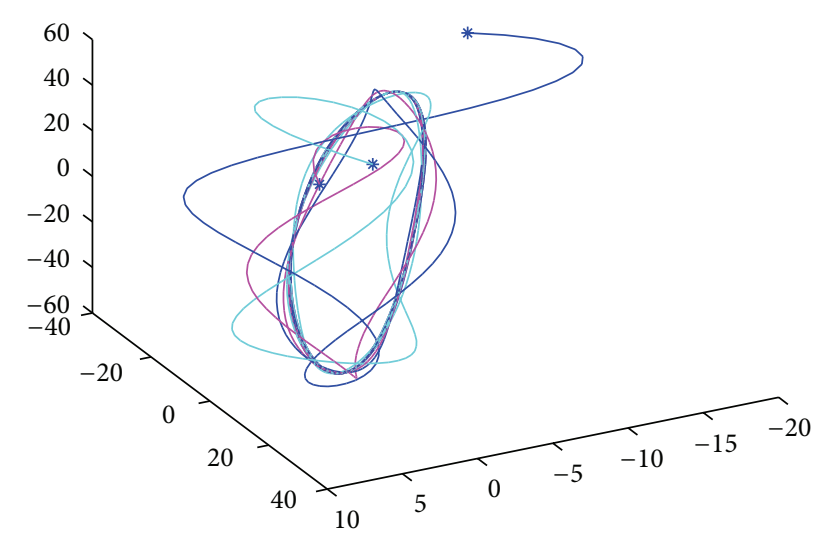

(a)

$\left[x_{i 1}(t), x_{i 2}(t), x_{i 3}\right], i=1,2,3$

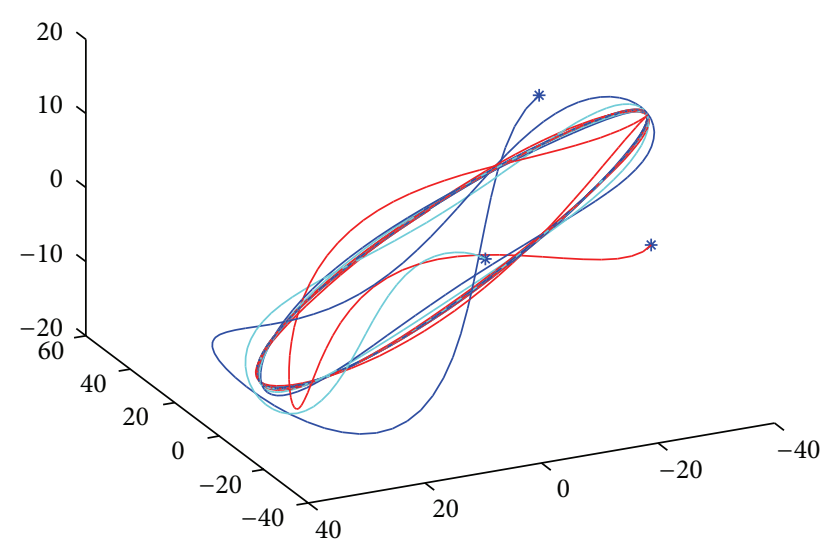

(b)

FIGURE 10: Trajectories of three agent's $\left[x_{i 1}, x_{i 2}, x_{i 3}, x_{i 4}, x_{i 5}, x_{i 6}\right]$ in the fifth instance with admissible bounded initial.

critically stable and the system matches the conditions of Theorem 25. It is asymptotically swarm stable and they achieve consensus with the consensus function is time varying. Figure 10, shows the trajectories of the $i$ th agent's state $\left[x_{i 1}, x_{i 2}, x_{i 3}, x_{i 4}, x_{i 5}, x_{i 6}\right]^{T} . *$ is the starting positions of agents.

\section{Conclusions}

The swarm stability problem of high-order LTI singular multiagent systems was solved through a method based on generalized inverse theory. Consensus is regarded as a specific type of swarm stability. The model considered in this paper is more general than the one in [18], and most of LTI homogeneous normal or singular multiagent system models appeared in the literature are specific cases of this one. A necessary and sufficient condition for swarm stability was presented via analyzing of the solution of the singular multiagents systems with the Drazin inverse theory. Swarm stability is a kind of nonequilibrium stability. Meanwhile Simulation results for reaching consensus are presented. From the simulation we can get that if the agent system can accept nonadmissible initial bounded value, the multiagent systems can be swarm stability with any initial bounded value.

\section{References}

[1] W. K. Potts, "The chorus-line hypothesis of manoeuvre coordination in avian flocks," Nature, vol. 309, no. 5966, pp. 344-345, 1984.

[2] J. K. Parrish, S. V. Viscido, and D. Grünbaum, "Self-organized fish schools: an examination of emergent properties," Biological Bulletin, vol. 202, no. 3, pp. 296-305, 2002.

[3] T. Vicsek, A. Czirok, E. B. Jacob, I. Cohenand, and O. Schochet, "Novel typeof phase transitions in a system of selfdrivenparticles," Physical Review Letters, vol. 75, pp. 1226-1229, 1995.

[4] A. Abdessameud and A. Tayebi, "Attitude synchronization of a group of spacecraft without velocity measurements," IEEE Transactions on Automatic Control, vol. 54, no. 11, pp. 26422648, 2009.

[5] R. Olfati-Saber, "Global configuration stabilization for the VTOL aircraft with strong input coupling," IEEE Transactions on Automatic Control, vol. 47, no. 11, pp. 1949-1952, 2002.

[6] J. A. Fax and R. M. Murray, "Information flow and cooperative control of vehicle formations," IEEE Transactions on Automatic Control, vol. 49, no. 9, pp. 1465-1476, 2004.

[7] R. Olfati-Saber, "Flocking for multi-agent dynamic systems: algorithms and theory," IEEE Transactions on Automatic Control, vol. 51, no. 3, pp. 401-420, 2006.

[8] P. Ögren, E. Fiorelli, and N. E. Leonard, "Cooperative control of mobile sensor networks: adaptive gradient climbing in a distributed environment," IEEE Transactions on Automatic Control, vol. 49, no. 8, pp. 1292-1302, 2004.

[9] K. Jin, P. Liang, and G. Beni, "Stability of synchronized distributed control of discrete swarm structures," in Proceedings of the IEEE International Conference on Robotics and Automation, pp. 1033-1038, San Diego, Calif, USA, May 1994.

[10] S. Ma, S. Hackwood, and G. Beni, "Multi-agent supporting systems (MASS): control with centralized estimator of disturbance," in Proceedings of the IEEE/RSJ/GI International Conference on Intelligent Robots and Systems, Advanced Robotic Systems and the Real World (IROS '94), pp. 679-686, Munich, Germany.

[11] Y. Liu, K. M. Passino, and M. Polycarpou, "Stability analysis of one-dimensional asynchronous swarms," IEEE Transactions on Automatic Control, vol. 48, no. 10, pp. 1848-1854, 2003.

[12] Y. Liu, K. M. Passino, and M. M. Polycarpou, "Stability analysis of $M$-dimensional asynchronous swarms with a fixed communication topology," IEEE Transactions on Automatic Control, vol. 48, no. 1, pp. 76-95, 2003.

[13] V. Gazi and K. M. Passino, "A class of attraction/repulsion functions for stable swarm aggregations," in Proceedings of the 41st IEEE Conference on Decision and Control, pp. 2842-2847, Las Vegas, Nev, USA, December 2002.

[14] V. Gazi and K. M. Passino, "Stability analysis of swarms," IEEE Transactions on Automatic Control, vol. 48, no. 4, pp. 692-697, 2003.

[15] V. Gazi, "Stability of an asynchronous swarm with timedependent communication links," IEEE Transactions on Systems, Man, and Cybernetics B, vol. 38, no. 1, pp. 267-274, 2008. 
[16] T. Chu, L. Wang, and T. Chen, "Self-organized motion in anisotropic swarms," Journal of Control Theory and Applications, vol. 1, no. 1, pp. 77-81, 2003.

[17] W. Li, "Stability analysis of swarm with general topology," IEEE Transactions on Systems, Man, and Cybernetics B, vol. 38, no. 4, pp. 1084-1097, 2008.

[18] N. Cai, J.-X. Xi, and Y.-S. Zhong, "Swarm stability of high-order linear time-invariant swarm systems," IET Control Theory \& Applications, vol. 5, no. 2, pp. 402-408, 2011.

[19] D. Swaroop, "Stability analysis of swarms with general topology," IEEE Transactions on Systems, Man, and Cybernetics B, vol. 4, no. 4, pp. 1084-1097, 2008.

[20] A. Pant, P. Seiler, and J. k. Hedrick, "String stability for a class of nonlinear systems," IEEE Transactions on Automatic Control, vol. 47, no. 2, pp. 349-357, 1996.

[21] L. Zhou, D. W. C. Ho, and G. S. Zhai, "Stability analysis of switched linear singular systems," Automatica, vol. 49, no. 5, pp. 1481-1487, 2013.

[22] A. Jadbabaie, J. Lin, and A. S. Morse, "Coordination of groups of mobile autonomous agents using nearest neighbor rules," IEEE Transactions on Automatic Control, vol. 48, no. 6, pp. 988-1001, 2003.

[23] R. Olfati-Saber and R. M. Murray, "Consensus problems in networks of agents with switching topology and time-delays," IEEE Transactions on Automatic Control, vol. 49, no. 9, pp. 1520 1533, 2004.

[24] W. Ren and R. W. Beard, "Consensus seeking in multiagent systems under dynamically changing interaction topologies," IEEE Transactions on Automatic Control, vol. 50, no. 5, pp. 655661, 2005.

[25] F. Xiao, L. Wang, and A. Wang, "Consensus problems in discrete-time multiagent systems with fixed topology," Journal of Mathematical Analysis and Applications, vol. 322, no. 2, pp. 587-598, 2006.

[26] P. Lin and Y. Jia, "Consensus of second-order discrete-time multi-agent systems with nonuniform time-delays and dynamically changing topologies," Automatica, vol. 45, no. 9, pp. 21542158, 2009.

[27] W. Yu, G. Chen, and M. Cao, "Some necessary and sufficient conditions for second-order consensus in multi-agent dynamical systems," Automatica, vol. 46, no. 6, pp. 1089-1095, 2010.

[28] Y.-P. Tian and Y. Zhang, "High-order consensus of heterogeneous multi-agent systems with unknown communication delays," Automatica, vol. 48, no. 6, pp. 1205-1212, 2012.

[29] Y. Zheng and L. Wang, "Finite-time consensus of heterogeneous multi-agent systems with and without velocity measurements," Systems \& Control Letters, vol. 61, no. 8, pp. 871-878, 2012.

[30] F. Xiao and L. Wang, "Consensus problems for highdimensional multi-agent systems," IET Control Theory and Applications, vol. 1, no. 3, pp. 830-837, 2007.

[31] L. Cheng, Z.-G. Hou, Y. Lin, M. Tan, and W. Zhang, "Solving a modified consensus problem of linear multi-agent systems," Automatica, vol. 47, no. 10, pp. 2218-2223, 2011.

[32] J. Xi, N. Cai, and Y. Zhong, "Consensus problems for high-order linear time-invariant swarm systems," Physica A, vol.389, no. 24, pp. 5619-5627, 2010.

[33] L. Dai, Singular Control Systems, vol. 118 of Lecture Notes in Control and Information Sciences, Springer, Berlin, Germany, 1989.

[34] X.-R. Yang and G.-P. Liu, "Necessary and sufficient consensus conditions of descriptor multi-agent systems," IEEE Transactions on Circuits and Systems, vol. 59, no. 11, pp. 2669-2677, 2012.
[35] J. X. Xi, Z. Y. Shi, and Y. S. Zhong, "Admissible consensus and consensualization of high order linear time-invariant singular swarm systems," Physica A, vol. 391, pp. 5839-5849, 2012.

[36] X. Dong, J. Xi, G. Lu, and Y. Zhong, "containment analysis and design for high-order linear time- invariant singular swarm systems with time delays," International Journal of Robust and Nonlinear Control, vol. 01, 2012.

[37] J. Xi, F. Meng, Z. Shi, and Y. Zhong, "Delay-dependent admissible consensualization for singular time-delayed swarm systems," Systems \& Control Letters, vol. 61, no. 11, pp. 1089-1096, 2012.

[38] M. P. Drazin, "Pseudo-inverses in associative rings and semigroups," The American Mathematical Monthly, vol. 65, pp. 506514, 1958.

[39] S. L. Campbell and C. D. Meyer, Generalized Inverse of Linear Transformations, Pitman, London, 1979, SIAM, Philadelphia, Pa, USA, 2009.

[40] S. L. Campbell, C. D. Meyer, and N. J. Rose, "Applications of the Drazin inverse to linear systems of differential equations with singular constant coefficients," SIAM Journal on Applied Mathematics, vol. 31, no. 3, pp. 411-425, 1976.

[41] C. Bu, K. Zhang, and J. Zhao, "Representations of the Drazin inverse on solution of a class singular differential equations," Linear and Multilinear Algebra, vol. 59, no. 8, pp. 863-877, 2011.

[42] J. Zhou, C. Bu, and Y. Wei, "Some block matrices with signed Drazin inverses," Linear Algebra and Its Applications, vol. 437, no. 7, pp. 1779-1792, 2012.

[43] C. Bu, C. Feng, and S. Bai, "Representations for the Drazin inverses of the sum of two matrices and some block matrices," Applied Mathematics and Computation, vol. 218, no. 20, pp. 10226-10237, 2012.

[44] C. Bu, J. Zhao, and J. Tang, "Representation of the Drazin inverse for special block matrix," Applied Mathematics and Computation, vol. 217, no. 10, pp. 4935-4943, 2011.

[45] J. Wang, D. Cheng, and X. Hu, "Consensus of multi-agent linear dynamic systems," Asian Journal of Control, vol. 10, no. 2, pp. 144-155, 2008.

[46] C. Meyer, Matrix Analysis and Applied Linear Algebra, SIAM, Philadelphia, Pa, USA, 2000.

[47] D. Cobb, "Controllability, observability, and duality in singular systems," IEEE Transactions on Automatic Control, vol. 29, no. 12, pp. 1076-1082, 1984.

[48] G. R. Duan, H. H. Yu, A. G. Wu, and X. Zhang, Analysis and Design of Descriptor Linear Systems, vol. 23 of Advances in Mechanics and Mathematics, Springer, New York, NY, USA, 2010. 


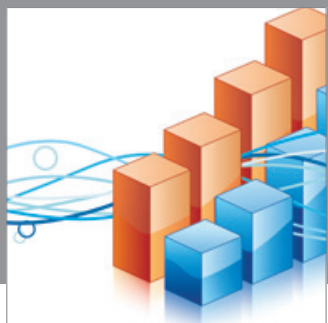

Advances in

Operations Research

mansans

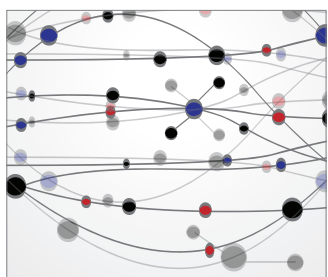

The Scientific World Journal
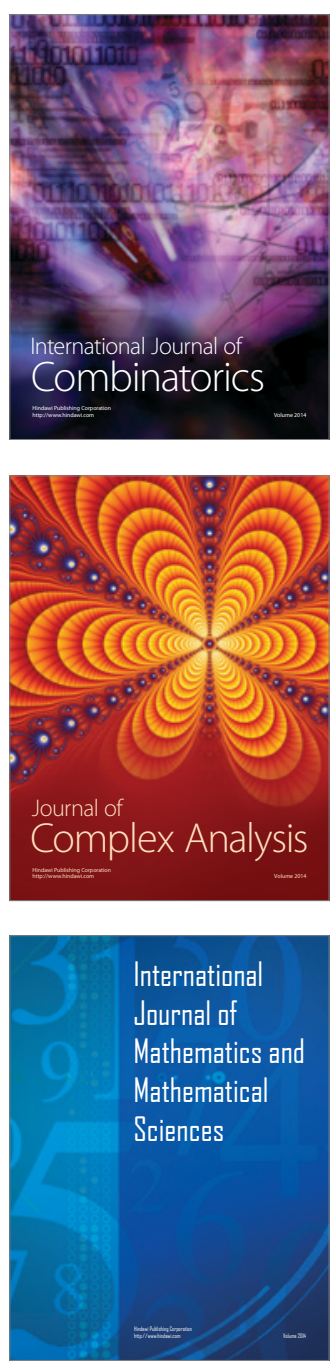
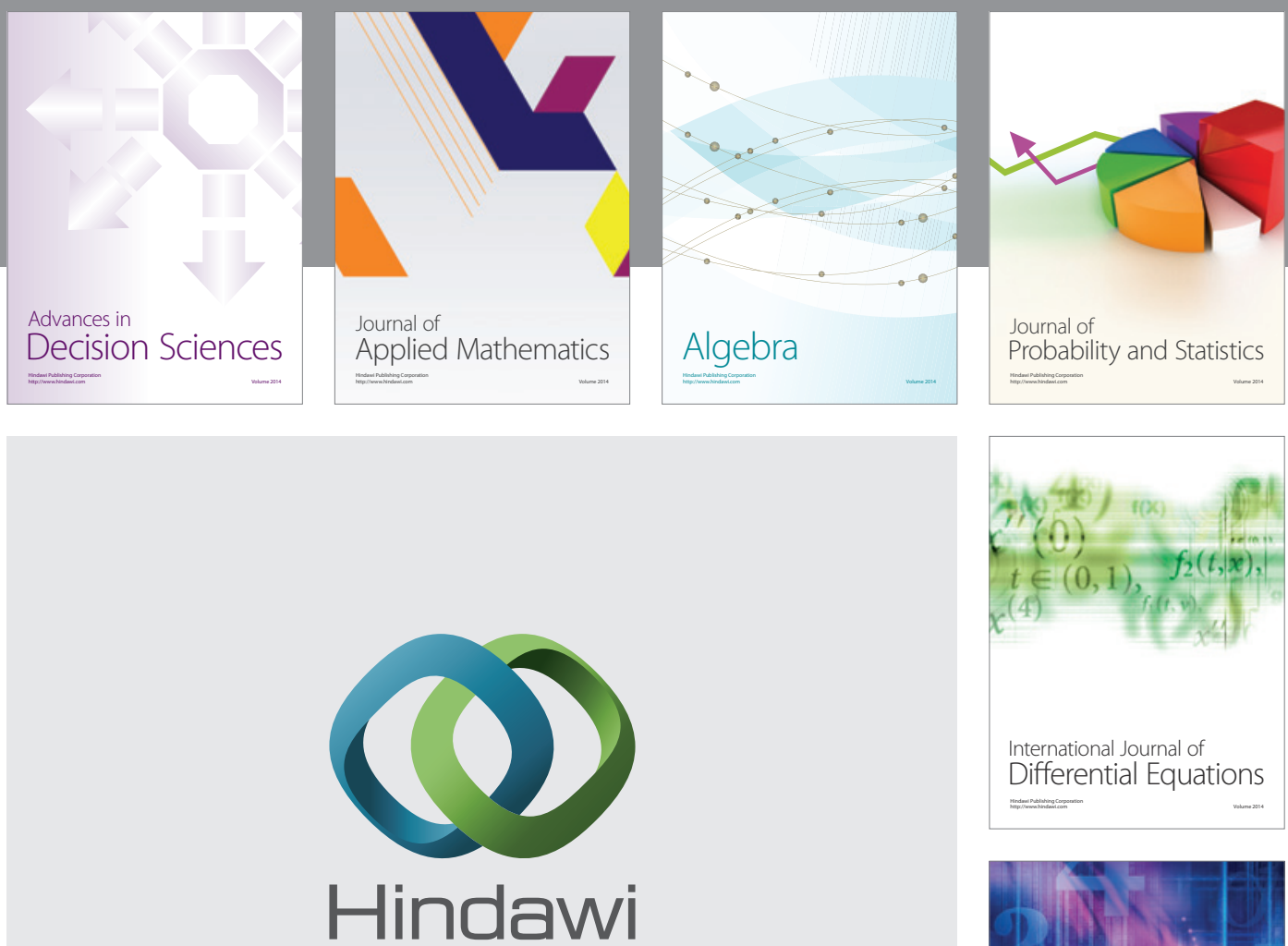

Submit your manuscripts at http://www.hindawi.com
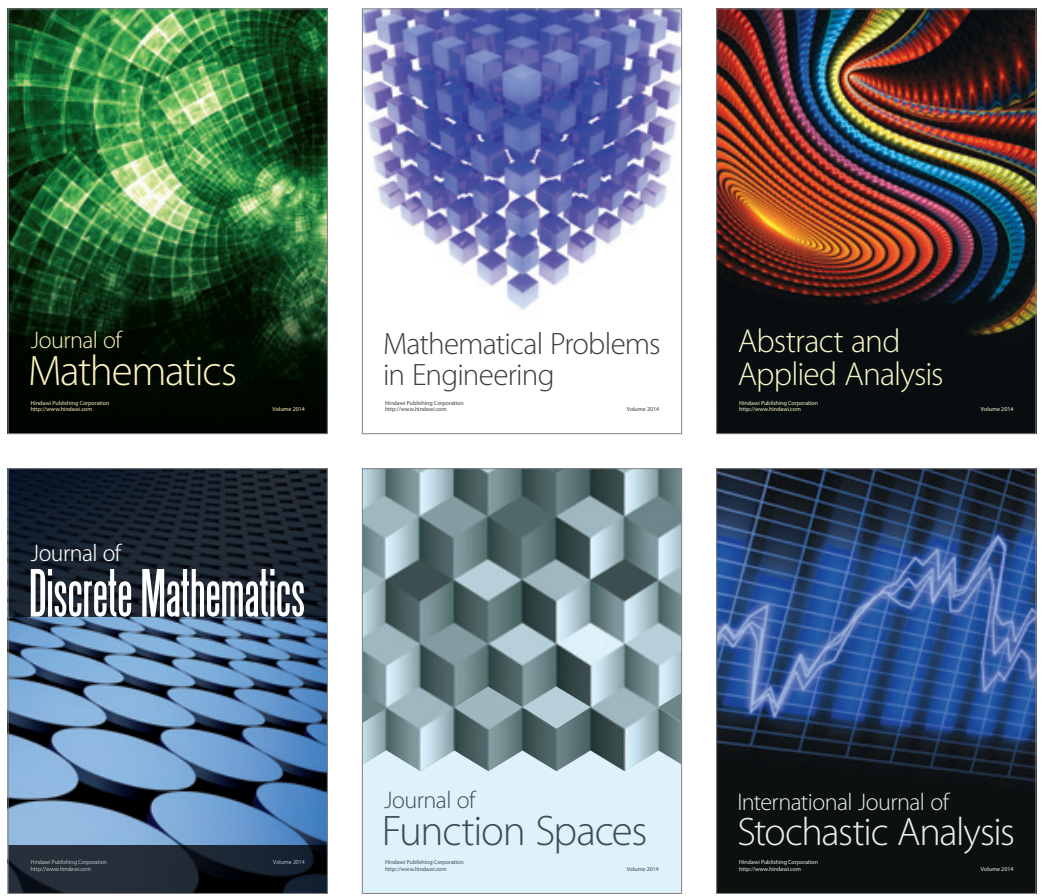

Journal of

Function Spaces

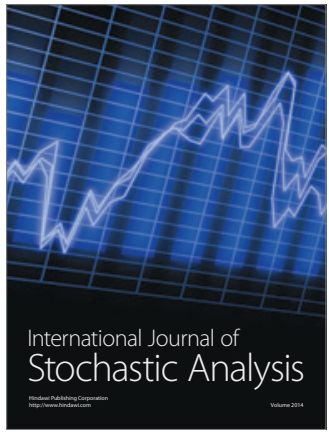

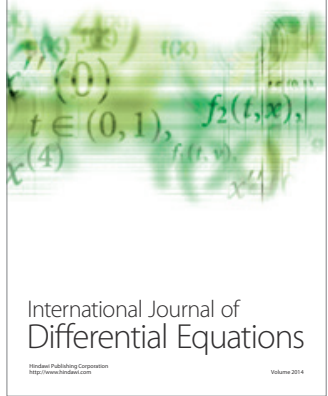
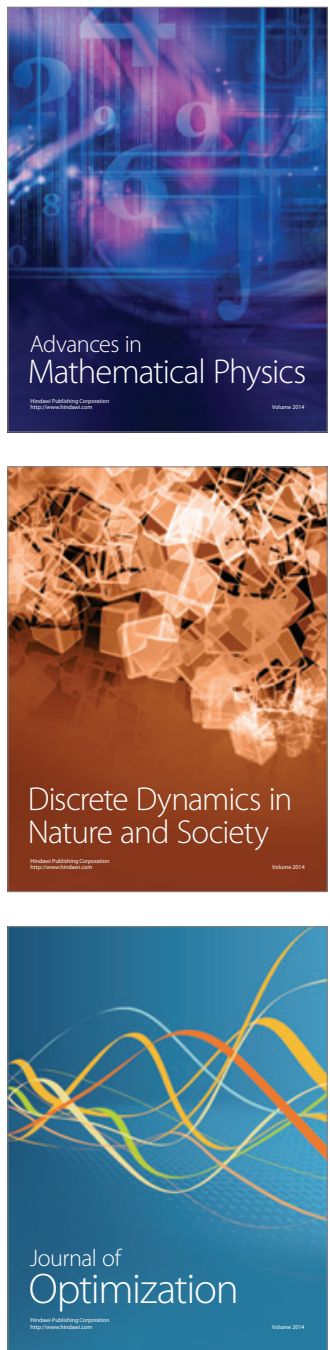